\title{
EQUIVARIANT ENDOMORPHISMS OF THE SPACE OF CONVEX BODIES
}

\author{
BY \\ ROLF SCHNEIDER
}

\begin{abstract}
We consider maps of the set of convex bodies in $d$-dimensional Euclidean space into itself which are linear with respect to Minkowski addition, continuous with respect to Hausdorff metric, and which commute with rigid motions. Examples constructed by means of different methods show that there are various nontrivial maps of this type. The main object of the paper is to find some reasonable additional assumptions which suffice to single out certain special maps, namely suitable combinations of dilatations and reflections, and of rotations if $d=2$. For instance, we determine all maps which, besides having the properties mentioned above, commute with affine maps, or are surjective, or preserve the volume. The method of proof consists in an application of spherical harmonics, together with some convexity arguments.
\end{abstract}

1. Introduction. Results. The set $\AA^{d}$ of all convex bodies (nonempty, compact, convex point sets) of $d$-dimensional Euclidean vector space $E^{d}(d \geq 2)$ is usually equipped with two geometrically natural structures: with Minkowski addition, defined by

$$
K_{1}+K_{2}=\left\{x_{1}+x_{2} \mid x_{1} \in K_{1}, x_{2} \in K_{2}\right\}, \quad K_{1}, K_{2} \in \Re^{d},
$$

and with the topology which is induced by the Hausdorff metric $\rho$, where

$$
\rho\left(K_{1}, K_{2}\right)=\inf \left\{\lambda \geq 0 \mid K_{1} \subset K_{2}+\lambda B, K_{2} \subset K_{1}+\lambda B\right\}, \quad K_{1} K_{2} \in \Re^{d} ;
$$

here $B=\left\{x \in E^{d} \mid\|x\| \leq 1\right\}$ denotes the unit ball. We wish to study the transformations of $\AA^{d}$ which are compatible with these structures, i.e., the continuous maps $\Phi: \Re^{d} \rightarrow \AA^{d}$ which satisfy $\Phi\left(K_{1}+K_{2}\right)=\Phi K_{1}+\Phi K_{2}$ for $K_{1}$, $K_{2} \in \Re^{d}$. However, from a geometrical point of view only those mappings seem to be of interest which commute with rigid motions applied to the bodies. Thus we are led to the following definition, where $M(d)$ denotes the group of proper rigid motions of $E^{d}$.

Definition. An endomorphism of the space $\Re^{d}$ is a map $\Phi: \Re^{d} \rightarrow \Re^{d}$ which satisfies (1.1) $\Phi\left(K_{1}+K_{2}\right)=\Phi K_{1}+K_{2}$ for $K_{1}, K_{2} \in \mathbb{\Re}^{d}$;

(1.2) $\Phi$ is continuous;

(1.3) $\Phi g=g \Phi$ for $g \in M(d)$.

Received by the editors April 27, 1973.

AMS (MOS) subject classifications (1970). Primary 52A20.

Key words and phrases. Convex body, Minkowski addition, Hausdorff metric, motion group, equivariant map, support function, mean width, Steiner point, characterization of functionals defined on the set of convex bodies, quermassintegral, convex function, spherical harmonic, multiplier. 
Property (1.1) is also expressed by saying that $\Phi$ is additive (in the sense of Minkowski), and property (1.3) is called the $M(d)$-equivariance of $\Phi$.

As an easy consequence of properties (1.1) and (1.2) we note that

$$
\Phi(\lambda K)=\lambda \Phi K \text { for } K \in \AA^{d} \text { and } \lambda \geq 0,
$$

where $\lambda K=\{\lambda x \mid x \in K\}$. Later we shall make use of this remark without further mention.

A problem concerned with certain endomorphisms of $\AA^{d}$ was apparently first posed by Grünbaum [13, p. 239]: After noting that there does not exist an additive map from $\Omega^{2}$ into $E^{2}$ which is affine-equivariant, i.e., commutes with affine transformations of $E^{2}$, he asks whether there is such a map from $\AA^{2}$ into the family of subsets of $E^{2}$, which is not the identical map. One example is given by the map $K \mapsto K+D K$, where $D K=K+(-K)$ denotes the difference set of $K$; here $-K$ is the image of $K$ under reflection in the origin of $E^{d}$, i.e. $-K$ $=\{-x \mid x \in K\}$. There are also more sophisticated examples: For $K \in \Omega^{2}$ let $S_{1}, S_{2}, \ldots$ be the finite (possibly empty) or infinite sequence (in any order) of the segments which are contained in the boundary of $K$. Let $S_{i}^{\prime}$ be the translate of $S_{i}$ which has its centre at the origin of $E^{2}$. Then it is easy to see that $S K=S_{1}^{\prime}+S_{2}^{\prime}+\cdots$ (with the obvious definition of the sum if it infinite) is a well-defined convex body and that the map $K \mapsto K+S K$ from $\Re^{2}$ into itself is additive and affine-equivariant. Evidently this map is not continuous. It is easy to see that the images under an additive, affine-equivariant map from $\Re^{d}$ into the subsets of $E^{d}$ are necessarily convex (Valette [27]). Under the additional assumption of continuity we have the following complete description of the maps in question.

(1.4) Theorem.(1) The affine-equivariant endomorphisms of $\AA^{d}$ are precisely the maps

$$
K \mapsto K+\lambda[K+(-K)], \quad K \in \Re^{d},
$$

where $\lambda \geq 0$ is a real constant.

We shall deduce Theorem (1.4) after having established some facts about the endomorphisms of $\AA^{d}$ in general. It would be nice to see a direct, more elementary proof of (1.4). Recently Valette [27] has investigated the continuous affine-equivariant maps $F: \Omega^{d} \rightarrow \Omega^{d}$ which, in lieu of (1.1), satisfy $F\left(K_{1}+K_{2}\right)$ $\supset F\left(K_{1}\right)+F\left(K_{2}\right)$.

The study of endomorphisms of $\Re^{d}$ is related to some questions and results of a similar type in the theory of convex bodies, which we are going to explain.

Let $u \cdot v$ denote the inner product of the vectors $u, v \in E^{d}$. The support function $H_{K}$ of a convex body $K \in \Re^{d}$ is defined by

(1) See the note after $\$ 7$. 


$$
H_{K}(u)=\max \{x \cdot u \mid x \in K\}, \quad u \in E^{d} .
$$

By the mean width $\beta(K)$ of a convex body $K \in \Re^{d}$ one understands the mean value over the unit sphere $S^{d-1}=\left\{u \in E^{d} \mid\|u\|=1\right\}$ of its width function $u \mapsto H_{K}(u)+H_{K}(-u)$, or

$$
\beta(K)=2 \omega_{d}^{-1} \int_{S^{d-1}} H_{K} d \omega .
$$

Here $\omega$ denotes Lebesgue measure on $S^{d-1}$, and $\omega_{d}=\omega\left(S^{d-1}\right)$ is the total area. The Steiner point $s(K)$ of $K \in \Re^{d}$ may be defined by means of the equation

$$
s(K)=d \omega_{d}^{-1} \int_{S^{d-1}} H_{K}(u) u d \omega(u)
$$

Evidently, the mappings $\beta: \Re^{d} \rightarrow \mathbf{R}$ and $s: \Re^{d} \rightarrow E^{d}$ are additive and continuous; $\beta$ is invariant and $s$ is equivariant with respect to rigid motions. Essentially, these properties suffice to characterize $\beta$ and $s$, respectively: If $\varphi: \Re^{d} \rightarrow \mathbf{R}$ is an additive, continuous, and motion-invariant map, then $\varphi(K)=\alpha \beta(K)$ for all $K$ $\in \Re^{d}$, where $\alpha$ is a real constant. This theorem is due to Hadwiger [15, p. 213]. Similarly: If $f: \AA^{d} \rightarrow E^{d}$ is an additive, continuous, and motion-equivariant map, then $f=s$. This has been proved, for $d=2$, by Shephard [26], and for general $d$ by Schneider [22]. A bit earlier, Meyer [18] had obtained the result under the stronger assumption of uniform continuity (with respect to the Hausdorff metric on $\AA^{d}$ ). Berg [2] has given a proof for $d=2$ and $d=3$ which allows us to weaken the continuity assumption. Sallee [20] has constructed a counterexample which shows that it is not possible to drop the continuity assumption entirely (compare also $\$ 7$ below).

It should be mentioned that these uniqueness results for the mean width and for the Steiner point are the key theorems for a series of further characterizations of real-valued and vector-valued functionals defined on $\Omega^{d}$. Such characterization theorems have been proved for the quermassintegrals by Hadwiger [15, (6.1.10)], and for certain closely related vector-valued functions (representing centroids of mass distributions defined by curvature functions) by Hadwiger-Schneider [16] and by Schneider [24].

After considering the map $\beta$ which associates with every convex body $K$ a real number with certain properties, and the map $s$ which associates with $K$ a certain point with similar properties, it seems natural to go one step further and to consider maps from $\Re^{d}$ into $\AA^{d}$ with formally the same properties, that is endomorphisms of $\Re^{d}$. Examples (besides $K \mapsto\{s(K)\}$ ) are near at hand: Let $B_{\alpha}(K)$ be the ball whose centre is the Steiner point of the convex body $K$ and whose radius is the mean width of $K$, multiplied by the positive real constant $\alpha$. It follows from the properties of the maps $\beta$ and $s$ that $\Phi K=B_{\alpha}(K)$ defines an endomorphism $\Phi$ of $\AA^{d}$. It has been asked in the book of Grünbaum [14, p. 135] whether every endomorphism of $\Re^{d}$ which in addition satisfies an Euler-type 
equation when restricted to polytopes, must be of this special kind. However, it has turned out that the additional assumption is not a true restriction, since in view of a result of Shephard [25, (18)] the validity of an Euler-type equation is already implied by additivity, together with reflection equivariance, and each endomorphism of $\AA^{d}$ commutes also with reflections, as will be clear from later considerations.

As a slightly more general example of an endomorphism of $\Re^{d}$ we mention the map $\Phi$ defined by

$$
\Phi K=\alpha_{1}[K-s(K)]+\alpha_{2}[-K+s(K)]+s(K)+\alpha \beta(K) B,
$$

where $\alpha_{1}, \alpha_{2}, \alpha$ are nonnegative real constants. For $d=2$, the constants $\alpha_{i}$ may be replaced by $\alpha_{i} g_{i}$, where $g_{i} \in S O(2)(i=1,2 ; S O(d)$ denotes the group of proper rotations of $\left.E^{d}\right)$. These endomorphisms will be called trivial. $\$ 2$ and part of $\$ 4$ is devoted to the construction of fairly large classes of nontrivial examples. Especially in the case $d=2$ we shall describe, for a given convex set $K \in \AA^{2}$, a rather general family of convex bodies which can occur as images of $K$ under some endomorphism of $\AA^{2}$. The following result, however, restricts the variety of conceivable endomorphisms considerably.

(1.7) Theorem. Every endomorphism of $\Re^{d}$ is uniquely determined by its value at one suitably chosen convex body, e.g. at a triangle with at least one irrational angle.

Here by an irrational angle we understand one which is an irrational multiple of $\pi$.

The generality of the examples mentioned above calls for reasonable additional assumptions under which an endomorphism of $\Re^{d}$ must be trivial. In this sense we have, besides (1.4), the following results. For their proof, Theorem (1.7) will be a useful tool.

(1.8) Theorem. Let $\Phi$ be an endomorphism of $\AA^{d}$.

(a) If the image under $\Phi$ of some at least one-dimensional convex body is zerodimensional, then

$$
\Phi K=\{s(K)\} \text { for } K \in \Re^{d} .
$$

(b) If the image under $\Phi$ of some convex body is a segment, then for each $K \in \AA^{d}$

$$
\Phi K=\lambda[K-s(K)]+\mu[-K+s(K)]+s(K)
$$

with real numbers $\lambda, \mu \geq 0(\lambda+\mu>0)$ if $d \geq 3$, and

$$
\Phi K=\lambda g[K-s(K)]+\mu g[-K+s(K)]+s(K)
$$

with $\lambda, \mu \geq 0(\lambda+\mu>0)$ and $g \in S O(2)$ if $d=2$. 
As consequences we have (1.4) and the following results.

(1.9) Corollary. The only surjective endomorphisms of $\Re^{d}$ are given by

$$
\Phi K=\lambda g[K-s(K)]+s(K) \text { for } K \in \mathbb{\Re}^{d},
$$

with $\lambda>0$, where $g$ is the identity or reflection in the origin if $d \geq 3$, and where $g \in S O(2)$ if $d=2$.

(1.10) Corollary. If the endomorphism $\Phi$ of $\AA^{d}$ is periodic, i.e. satisfies $\Phi^{n} K=K$ for some positive integer $n$ (which may depend on $K$ ) and for each $K \in \Re^{d}$, then

$$
\Phi K=g[K-s(K)]+s(K) \text { for } K \in{\Re^{d}}^{d},
$$

where $g \in\{ \pm$ identity $\}$ if $d \geq 3$, and where $g \in S O(2)$ is a rotation by a rational angle, if $d=2$.

(1.12) Corollary. If the endomorphism $\Phi$ of $\AA^{d}$ maps $d$-polytopes, into polytopes, then $\Phi$ is the map described in $(1.8)(\mathrm{b})$, provided that $d \geq 3$.

Let $W_{k}(K)$ denote the $k$ th quermassintegral of $K\left(W_{0}\right.$ is the volume). If

(1.13) Corollary. Let $\Phi$ be an endomorphism of $\Re^{d}$, and let $k \in\{0,1, \ldots, d-2\}$.

$$
W_{k}(\Phi K)=W_{k}(K) \text { for } K \in{\AA^{d}}^{d}
$$

then $\Phi$ is the map given by (1.11) with $g \in\{ \pm$ identity $\}$, respectively $g \in S O(2)$.

The content of the subsequent sections is as follows. In $\$ 2$ we construct a class of nontrivial endomorphisms of $\Re^{d}$. Another way of constructing such examples is described in §4. These different examples are treated with some detail since the problem of characterizing explicitly all endomorphisms of $\Re^{d}$ is unsolved, and a knowledge of various examples might prove useful in further study of this question. In $\$ 3$ we collect some auxiliary results on spherical harmonics and support functions which will be needed later. In $\$ 4$ spherical harmonics are used to give a certain description of endomorphisms of $\AA^{d}$, which will be the basis for the rest of the paper. The proof of (1.7) follows in $\$ 5$, and the other results are proved in $\S 6$. Finally, $\S 7$ contains examples of maps $\Phi: \AA^{d} \rightarrow \AA^{d}$ which satisfy (1.1) and (1.3), but not (1.2).

As to the general nature of the following considerations, one might say that the method of approach consists in a combination of elementary facts from harmonic analysis with convexity arguments.

Notation. The following symbols are used in the paper. Part of them have already been defined above; the remaining ones will be explained in later sections (if necessary). 
$\mathbf{R} \quad$ set of real numbers

$E^{d} \quad d$-dimensional Euclidean vector space

$a \cdot b \quad$ scalar product of vectors $a, b \in E^{d}$

$\|a\| \quad$ length of vector $a \in E^{d}$

$B \quad=\left\{x \in E^{d} \mid\|x\| \leq 1\right\}$, unit ball of $E^{d}$

$S^{d-1} \quad=\left\{x \in E^{d} \mid\|x\|=1\right\}$, unit sphere of $E^{d}$

$\omega \quad$ Lebesgue measure on $S^{d-1}$

$\omega_{d} \quad=\omega\left(S^{d-1}\right)$, total surface area of the unit sphere in $E^{d}$

$S O(d) \quad$ proper orthogonal group of $E^{d}$

$M(d) \quad$ group of proper rigid motions of $E^{d}$

$\Re^{d} \quad$ set of all convex bodies in $E^{d}$

$H_{K} \quad$ support function of $K \in \AA^{d}$

$\beta(K) \quad$ mean width of $K \in \AA^{d}$

$s(K) \quad$ Steiner point of $K \in \AA^{d}$

$W_{k}(K) \quad k$ th quermassintegral of $K \in \AA^{d}$

$C\left(S^{d-1}\right)$ real vector space of real continuous functions on $S^{d-1}$

$\langle$,$\rangle \quad usual inner product on C\left(S^{d-1}\right)$

ङ $\sigma^{d} \quad$ cone of support functions (restricted to $S^{d-1}$ ) contained in $C\left(S^{d-1}\right)$

$C_{m}^{\prime} \quad$ ultraspherical polynomial of degree $m$ and order $\nu$

$\mathfrak{S}_{m}^{d} \quad$ subspace of $C\left(S^{d-1}\right)$ consisting of spherical harmonics of degree $m$

$\Pi_{m} \quad$ orthogonal projection from $C\left(S^{d-1}\right)$ to $\mathfrak{B}_{m}^{d}$

$\mathfrak{S}^{d} \quad$ subspace of $C\left(S^{d-1}\right)$ consisting of all finite linear combinations of spherical harmonics.

If $K \in \Re^{d}$ and $x \in E^{d}$, we often write $K+x$ instead of $K+\{x\}$. It will sometimes be convenient not to distinguish between a support function, which is defined on $E^{d}$, and its restriction to $S^{d-1}$; we use the same notation $H_{K}$, and the domain will always be clear from the context.

2. Examples of endomorphisms of $\AA^{d}$. In this section we shall establish the existence of many nontrivial endomorphisms of $\Re^{d}$. First we consider the case $d=2$. 
Let $\mu$ be a positive Borel measure on the group $S O(2)$. If we define

$$
H_{K}^{*}(u)=\int_{s O(2)} H_{K-s(K)}(g u) d \mu(g)+s(K) \cdot u, \quad u \in E^{2},
$$

for $K \in \Re^{2}$, then it is easy to see that $H_{K}^{*}$ satisfies the sufficient conditions for a support function; hence there is a unique convex body $\Phi K \in \Omega^{2}$ such that

$$
H_{\Phi K}=H_{K}^{*} \text {. }
$$

The map $\Phi: \Re^{2} \rightarrow \Re^{2}$ thus defined turns out to be an endomorphism of $\AA^{2}$.

This observation yields some insight into the freedom which one has in prescribing the image of a given convex set under an endomorphism. For a convex body $K$ in $E^{d}$ let $[K]_{0}$ denote the set of all elements of $\AA^{d}$ which can be approximated by bodies of the form $\lambda_{1} g_{1} K+\cdots+\lambda_{r} g_{r} K$, where $g_{1}, \ldots, g_{r}$ $\in S O(d)$ are rotations and $\lambda_{1}, \ldots, \lambda_{r}$ are positive real numbers; and let $[K]$ be the set of all translates of bodies of $[K]_{0}$. Thus, for instance, if $K$ is a segment, $[K]$ is the class of all "zonoids", which for $d=2$ coincides with the set of all centrally symmetric convex bodies.

(2.3) Proposition. Let $L \in \Omega^{2}$ be given, and choose $\bar{L} \in[L]$ such that the Steiner points of $L$ and $\bar{L}$ coincide. Then there exists an endomorphism $\Phi$ of $\Omega^{2}$ which satisfies $\Phi L=\bar{L}$.

Proof. Write $L_{0}=L-s(L)$ and $\bar{L}_{0}=\bar{L}-s(L)$. Then $\bar{L}_{0} \in\left[L_{0}\right]$, but since the Steiner points of $L_{0}$ and $\bar{L}_{0}$ both coincide with the origin of $E^{2}$, we have in fact $\bar{L}_{0} \in\left[L_{0}\right]_{0}$. Hence there exists a positive Borel measure $\mu$ on $S O(2)$ such that

$$
H_{\bar{L}_{0}}(u)=\int_{S O(2)} H_{L_{0}}(g u) d \mu(g), \quad u \in E^{2} .
$$

(See, e.g., Edwards [11, Theorem $2_{\alpha}$ ]. Observe that convergence of convex bodies is equivalent to the fact that the corresponding support functions converge uniformly on $S^{d-1}$.) Now use this measure $\mu$ in definition (2.1). Then $\Phi$ defined by (2.2) is an endomorphism of $\Omega^{2}$ which satisfies $\Phi L=\bar{L}$. We remark that in general $\Phi$ is not uniquely determined by the properties demanded in (2.3).

We do not know whether there exist endomorphisms of $\Omega^{2}$ other than those defined by (2.1), (2.2). It is, however, not difficult to prove that every uniformly continuous endomorphism of $\Omega^{2}$ must be of this convolution type, with some suitable measure $\mu$. Perhaps we should point out here that a map $\Phi: \Omega^{2} \rightarrow \AA^{2}$ which satisfies only (1.1) and (1.2) need not be uniformly continuous, as can be shown by counterexamples.

Now we consider the case $d \geq 3$. We cannot take just (2.1), with $S O$ (2) replaced by $S O(d)$, since generally the map thus defined would not commute with rotations. The proper extension to higher dimensions is as follows. Let $f$ be (for simplicity) a continuous real function, defined on the interval $[-1,1]$, and for 
$K \in \Re^{d}$ define $H_{K}^{*}(0)=0$ and

$$
H_{K}^{*}(u)=\|u\| \int_{S^{d-1}} H_{K-s(K)}(v) f\left(\|u\|^{-1} u \cdot v\right) d \omega(v)+s(K) \cdot u, \quad, \quad u \in E^{d} \backslash\{0\} .
$$

Then we have $H_{g K}^{*}(u)=H_{K}^{*}(g u)$ for $u \in E^{d}$ and $g \in S O(d)$, and $H_{K+t}^{*}(u)$ $=H_{K}^{*}+t \cdot u$ for $u, t \in E^{d}$. Furthermore, $H_{K}^{*}$ is continuous and positively homogeneous of degree one. However, it is only for specially chosen nonnegative functions $f$ that we can prove convexity for the function $H_{K}^{*}$. One obvious possibility consists in choosing $f$ in such a manner that $u \mapsto\|u\| f\left(\|u\|^{-1} u \cdot v\right)$ is, for fixed $v$, a convex function on $E^{d} \backslash\{0\}$ (observe that $H_{K-s(K)} \geq 0$ ). More interesting is the following procedure which goes back, essentially, to an idea of Berg [1, pp. 10-12]. We take a function $q:[0, \infty) \rightarrow[0, \infty)$, for simplicity continuous and with compact support, and put

$$
\chi(u, v)=\int_{0}^{\infty} q(\|u-r v\|) r^{d} d r \quad \text { for } u, v \in S^{d-1} .
$$

Since $\|u-r v\|^{2}=1+r^{2}-2 r u \cdot v, \chi(u, v)$ depends only on $u \cdot v$, so that there is a nonnegative continuous real function $f$ on $[-1,1]$ with $\chi(u, v)=f(u \cdot v)$. Using this $f$ in definition (2.4), and making the inessential assumption that $s(K)=0$, we get for $u \in S^{d-1}$

$$
\begin{aligned}
H_{K}^{*}(u) & =\int_{S^{d-1}} H_{K}(v) f(u \cdot v) d \omega(v) \\
& =\int_{S^{d-1}} H_{K}(v)\left\{\int_{0}^{\infty} q(\|u-r v\|) r^{d} d r\right\} d \omega(v) \\
& =\int_{S^{d-1}} \int_{0}^{\infty} H_{K}(r v) q(\|u-r v\|) r^{d-1} d r d \omega(v) \\
& =\int_{E^{d}} H_{K}(z) q(\|u-z\|) d z \\
& =\int_{E^{d}} H_{K}(u-z) q(\|z\|) d z ;
\end{aligned}
$$

hence, for $x \in E^{d}$,

$$
\begin{aligned}
H_{K}^{*}(x) & =\int_{E^{d}} H_{K}(x-\|x\| z) q(\|z\|) d z \\
& =\frac{1}{2} \int_{E^{d}}\left\{H_{K}(x-\|x\| z)+H_{K}(x+\|x\| z)\right\} q(\|z\|) d z .
\end{aligned}
$$

(2.5) Proposition. Let $H$ be a support function on $E^{d}$, and let $z \in E^{d}$. Then the function $H_{2}$ defined by

$$
H_{z}(x)=H(x-\|x\| z)+H(x+\|x\| z), \quad x \in E^{d},
$$

is a support function. 
It is trivial that $H_{2}$ is continuous and positively homogeneous of degree one. The following proof of the convexity of $H_{z}$ (as well as the idea of splitting the integral giving $H_{K}^{*}(x)$ as above) is due to Wolfgang Weil. For $x, y \in E^{d}$ and arbitrary $\alpha \in[0,1]$ we may write

$$
\begin{aligned}
H_{z}(x+y)= & H(x+y-\|x+y\| z)+H(x+y+\|x+y\| z) \\
\leq & H(x-\alpha\|x+y\| z)+H(y-(1-\alpha)\|x+y\| z) \\
& +H(x+\alpha\|x+y\| z)+H(y+(1-\alpha)\|x+y\| z) .
\end{aligned}
$$

Without loss of generality we may assume that $x$ and $y$ are linearly independent. Put

$$
\alpha=\frac{\|x\|}{\|x\|+\|y\|}, \quad \beta=\frac{\|x+y\|}{\|x\|}, \quad \gamma=\frac{\|x+y\|}{\|y\|}
$$

then we have $1-\alpha \beta>0$ and $1-(1-\alpha) \gamma>0$. From

$$
\begin{aligned}
& 2(x-\alpha\|x+y\| z)=(1+\alpha \beta)(x-\|x\| z)+(1-\alpha \beta)(x+\|x\| z), \\
& 2(x+\alpha\|x+y\| z)=(1-\alpha \beta)(x-\|x\| z)+(1+\alpha \beta)(x+\|x\| z),
\end{aligned}
$$

and the convexity of $H$ it follows that

$$
\begin{aligned}
& H(x-\alpha\|x+y\| z)+H(x+\alpha\|x+y\| z) \\
& \leq H(x-\|x\| z)+H(x+\|x\| z)=H_{z}(x) .
\end{aligned}
$$

Analogously we obtain

$$
\begin{aligned}
H(y-(1-\alpha)\|x+y\| z) & +H(y+(1-\alpha)\|x+y\| z) \\
& \leq H(y-\|y\| z)+H(y+\|y\| z)=H_{z}(y) .
\end{aligned}
$$

Together with (2.6) this gives $H_{z}(x+y) \leq H_{z}(x)+H_{z}(y)$, which proves Proposition (2.5).

Since $q$ is nonnegative, we arrive at once at the conclusion that $H_{K}^{*}$ is convex. Hence there is a map $\Phi: \Re^{d} \rightarrow \Re^{d}$ such that $H_{\Phi K}=H_{K}^{*}$ for each $K \in \Omega^{d}$. Clearly, $\Phi$ is an endomorphism of $\AA^{d}$.

Of course, the whole construction could be generalized by introducing suitable measures. Thus generalized, formula (2.4) would represent all the uniformly continuous endomorphisms of $\Re^{d}$, as can be deduced from a result of Dunkl [10, p. 259]. However, the complete characterization of the measures occurring is an open question.

Let us briefly mention another possibility of constructing endomorphisms of $\Re^{d}$. For $K \in \Re^{d}$ let $\mu_{1}(K ; \cdot)$ denote the first surface area measure of $K$ as defined, e.g., by Fenchel-Jessen [12]. Let $f:[-1,1] \rightarrow \mathbf{R}$ be continuous, and for $K \in \Omega^{d}$ define $H_{K}^{*}(0)=0$ and 


$$
H_{K}^{*}(u)=\|u\| \int_{s^{d-1}} f\left(\|u\|^{-1} u \cdot v\right) d \mu_{1}(K ; v)+s(K) \cdot u, \quad u \in E^{d} \backslash\{0\} .
$$

If $f$ is suitably chosen, for instance such that $u \mapsto\|u\| f\left(\|u\|^{-1} u \cdot v\right)$ is convex on $E^{d} \backslash\{0\}$, the function $H_{K}^{*}$ will be a support function for each $K \in \Omega^{d}$. Define $\Phi: \Re^{d} \rightarrow \Omega^{d}$ by $H_{\Phi K}=H_{K}^{*}$. Then it follows from well-known properties of the measure $\mu_{1}$ that $\Phi$ is an endomorphism of $\Omega^{d}$.

3. Spherical harmonics and support functions. In order to prove the theorems formulated in the introduction we shall make use of spherical harmonics. In this section we list the necessary notions and some auxiliary results. For an introduction to the theory of spherical harmonics, where these facts can be found, the reader is referred to Müller [19], Coifman-Weiss [7], and the literature quoted there. Some special results pertaining to the representation of support functions by means of spherical harmonics, which will be needed later, are also proved in this section.

Let $C\left(S^{d-1}\right)$ denote the real vector space of real continuous functions on the sphere $S^{d-1}$, endowed with the maximum norm. On $C\left(S^{d-1}\right)$ the group $S O(d)$ of (orientation preserving) orthogonal linear transformations of $E^{d}$ operates by left translation; here the left translate $g F$ of an element $F \in C\left(S^{d-1}\right)$ by the rotation $g \in S O(d)$ is defined by $(g F)(u)=F\left(g^{-1} u\right)$ for $u \in S^{d-1}$. A linear subspace $V \subset C\left(S^{d-1}\right)$ is called invariant if it is closed and if $F \in V$ implies $g F \in V$ for all $g \in S O(d)$. An invariant subspace $V$ is called irreducible if it does not contain an invariant subspace different from $\{0\}$ and $V$. By definition, a spherical harmonic on $S^{d-1}$ is an element of an irreducible subspace of $C\left(S^{d-1}\right)$. Explicitly, a spherical harmonic of degree $m$ is (either identically zero or) the restriction to $S^{d-1}$ of a real function on $E^{d}$ which in Cartesian coordinates is expressed as a homogeneous harmonic polynomial of degree $m$. For $m=0,1,2, \ldots$ we denote by $\mathfrak{S}_{m}^{d}$ the space of all spherical harmonics on $S^{d-1}$ of degree $m$. Each space $\mathfrak{S}_{m}^{d}$ is irreducible. Its dimension is given by

$$
\operatorname{dim} \mathfrak{S}_{m}^{d}=\frac{2 m+d-2}{m+d-2}\left(\begin{array}{c}
m+d-2 \\
m
\end{array}\right) .
$$

In the case $d=2$ it is often convenient to describe a vector $u \in S^{d-1}$ by the angle $\varphi \in[0,2 \pi)$ determined by $u=e_{1} \cos \varphi+e_{2} \sin \varphi$ where $e_{1}, e_{2}$ is an orthonormal basis of $E^{2}$. We have $F \in \mathfrak{S}_{m}^{2}$ if and only if the function $f$ defined by $f(\varphi)=F(u)$ is of the form $f(\varphi)=a \cos m \varphi+b \sin m \varphi$ with real constants $a, b$.

If $p \in S^{d-1}$, the space $\mathfrak{S}_{m}^{d}$ contains functions which are rotationally symmetric with respect to $p$, i.e. functions $F$ of the form $F(u)=f(u \cdot p)$ for $u \in S^{d-1}$. Every such spherical harmonic $F$ is given by $F(u)=\alpha C_{m}^{v}(u \cdot p)$, where $\alpha$ is a real constant and

$$
C_{m}^{p}(t)=\sum_{j=0}^{[m / 2]} \frac{(-1)^{j}(\nu+m-j-1) !}{(\nu-1) !(m-2 j) ! j !}(2 t)^{m-2 j}
$$


denotes the ultraspherical (or Gegenbauer) polynomial of degree $m$ and order $\nu=\frac{1}{2}(d-2)$. It can also be represented by means of Rodrigues' formula

$$
C_{m}^{\nu}(t)=\alpha_{m}^{\nu}\left(1-t^{2}\right)^{-\nu+1 / 2} \frac{d^{m}}{d t^{m}}\left(1-t^{2}\right)^{m+\nu-1 / 2},
$$

where $\alpha_{m}^{\nu} \neq 0$ is a certain constant. The linear hull of the set of functions $\left\{u \mapsto C_{m}^{\nu}(u \cdot p) \mid p \in S^{d-1}\right\}$ is all of $\mathfrak{E}_{m}^{d}$.

By $\mathscr{G}^{d} \subset C\left(S^{d-1}\right)$ we denote the subspace of all finite linear combinations of spherical harmonics on $S^{d-1}$. It is dense in $C\left(S^{d-1}\right)$. The elements of $\mathfrak{G}^{d}$ are called generalized trigonometric polynomials on $S^{d-1}$.

If the vector space $C\left(S^{d-1}\right)$ is endowed with the inner product $\langle$,$\rangle defined by$

$$
\langle F, G\rangle=\int_{S^{d-1}} F G d \omega, \quad F, G \in C\left(S^{d-1}\right),
$$

then any two spherical harmonics of different degrees are orthogonal, i.e. $\langle F, G\rangle=0$ for $F \in \mathfrak{S}_{m}^{d}, G \in \mathfrak{S}_{n}^{d}, m \neq n$. By

$$
\Pi_{m}: C\left(S^{d-1}\right) \rightarrow \mathfrak{Q}_{m}^{d}
$$

we denote the orthogonal projection from $C\left(S^{d-1}\right)$ onto the subspace $\mathfrak{S}_{m}^{d}$ $(m=0,1,2, \ldots)$; thus if $Y_{m 1}, \ldots, Y_{m N}$ (where $\left.N=\operatorname{dim} \mathfrak{S}_{m}^{d}\right)$ is an orthonormal basis of $\mathfrak{Y}_{m}^{d}$,

$$
\Pi_{m} F=\sum_{j=1}^{N}\left\langle F, Y_{m j}\right\rangle Y_{m j}, \quad F \in C\left(S^{d-1}\right) .
$$

The "Addition Theorem"

$$
\sum_{j=1}^{N} Y_{m j}(u) Y_{m j}(v)=\beta_{m}^{p} C_{m}^{\nu}(u \cdot v), \quad u, v \in S^{d-1},
$$

where $\beta_{m}^{v} \neq 0$ is a suitable constant, leads to a representation of the projection which does not depend on the choice of a basis, namely

$$
\left(\Pi_{m} F\right)(u)=\beta_{m}^{v} \int_{S^{d-1}} C_{m}^{v}(u \cdot v) F(v) d \omega(v), \quad u \in S^{d-1} .
$$

It is obvious that $\Pi_{m}$ is linear and continuous with respect to the maximum norm. In view of (3.5) it is evident that the projection $\Pi_{m}$ commutes with rotations, i.e.

$$
\Pi_{m}(g F)=g\left(\Pi_{m} F\right), \quad g \in S O(d), F \in C\left(S^{d-1}\right) .
$$

For later applications we compute $\Pi_{m} F$ for a function $F \in C\left(S^{d-1}\right)$ with rotational symmetry, i.e. which is of the form $F(u)=f(u \cdot p)$ with some fixed vector $p \in S^{d-1}$. Formula (3.5) and the Funk-Hecke formula, that is

$$
\int_{S^{d-1}} f(u \cdot v) Y_{m}(v) d \omega(v)=\eta_{m}^{\nu} \lambda_{m}^{v}[f] Y_{m}(u), \quad u \in S^{d-1},
$$


where $Y_{m} \in \mathfrak{S}_{m}^{d}, \eta_{m}^{\gamma} \neq 0$ is a suitable constant, and

$$
\lambda_{m}^{\nu}[f]=\int_{-1}^{1} f(t) C_{m}^{\nu}(t)\left(1-t^{2}\right)^{r-1 / 2} d t,
$$

lead to

$$
\left(\Pi_{m} F\right)(u)=\beta_{m}^{\prime} \eta_{m}^{\prime} \lambda_{m}^{\prime}[f] C_{m}^{\prime}(u \cdot p), \quad u \in S^{d-1} .
$$

We are now going to collect some auxiliary results which will be needed in applying spherical harmonics to the study of support functions of convex bodies. If $K \in \Re^{d}$ is a convex body, then the restriction of its support function to $S^{d-1}$ is an element of $C\left(S^{d-1}\right)$. Accordingly, a function $F \in C\left(S^{d-1}\right)$ will be called a support function if its homogeneous extension defined by $F(x)=\|x\| F\left(\|x\|^{-1} x\right)$ for $x \in E^{d} \backslash\{0\}$ is convex. The set of all support functions on $S^{d-1}$ is a closed convex cone $\widetilde{\Im}^{d}$ in $C\left(S^{d-1}\right)$. If $K \in \Omega^{d}$, the support function of $K$ and its restriction to $S^{d-1}$ will be denoted by the same symbol $H_{K}$. In passing, we notice the following identities, which are immediate consequences of the definitions (1.4) and (1.5):

$$
\begin{gathered}
\left(\Pi_{0} H_{K}\right)(u)=\frac{1}{2} \beta(K) \\
\left(\Pi_{1} H_{K}\right)(u)=s(K) \cdot u \quad \text { for } u \in S^{d-1}, K \in \Omega^{d} .
\end{gathered}
$$

Whether a twice continuously differentiable function $F \in C\left(S^{d-1}\right)$ is a support function can be decided by means of a well-known criterion: Extend $F$ to $E^{d} \backslash\{0\}$ as above, and let $F_{i k}=\partial^{2} F / \partial x_{i} \partial x_{k}$, where $x_{1}, \ldots, x_{d}$ are Cartesian coordinates. By homogeneity, the matrix with elements $F_{i k}(x)$ has 0 as an eigenvalue. Let $\eta(F, x)$ denote the smallest of the remaining $d-1$ eigenvalues (with $\eta(F, x)=0$ if and only if the eigenvalue 0 has multiplicity greater than one); and let $\eta(F)$ be the minimum of $\eta(F, x)$ for $\|x\|=1$. As convexity of $F$ is equivalent to the condition that the quadratic form $\sum F_{i k}(x) \alpha_{i} \alpha_{k}$ be positive semidefinite for each $x \in E^{d} \backslash\{0\}$, we have the following criterion:

(3.11) The twice continuously differentiable function $F \in C\left(S^{d-1}\right)$ is a support function if and only if $\eta(F) \geq 0$.

The following two corollaries are easily deduced from (3.11).

(3.12) Every twice continuously differentiable function in $C\left(S^{d-1}\right)$ is the difference of two support functions.

In fact, if $F \in C\left(S^{d-1}\right)$ is twice continuously differentiable, then $\eta\left(F+H_{\rho B}\right)$ $=\eta(F)+\rho$ which is positive for sufficiently large $\rho$.

(3.13) If $F_{1}, \ldots, F_{k} \in C\left(S^{d-1}\right)$ are twice continuously differentiable functions, then there exists a positive constant $\sigma$ such that $1+\sum \alpha_{i} F_{i}$ is a support function for all choices of real numbers $\alpha_{1}, \ldots, \alpha_{k}$ satisfying $\sum \alpha_{i}^{2} \leq \sigma$. 
This follows at once from the fact that the eigenvalues of a matrix depend continuously on its entries.

Since every support function is continuous, it can be approximated, uniformly on $S^{d-1}$, by generalized trigonometric polynomials. We shall need the fact that these approximating functions can also be chosen as support functions; in other words:

(3.14) $\mathfrak{S}^{d} \cap \mathbb{\mho}^{d}$ is dense in $\mathfrak{\mho}^{d}$.

A proof has been given in [22]; however, we prefer the following one which does not make use of analyticity assumptions and series expansions. Let us first assume that $H \in \mho^{d}$ is twice continuously differentiable and satisfies $\eta(H)>0$. By an extended version of the Weierstrass approximation theorem (see, e.g., Courant-Hilbert $\left[9\right.$, p. 68]) there exists a sequence $P_{1}, P_{2}, \ldots$ of polynomials on $E^{d}$ such that the (extended) function $H$ and its derivatives up to the second order are approximated uniformly on, say, $\left\{x \in E^{d} \mid \frac{1}{2} \leq\|x\| \leq 2\right\}$ by the polynomials and their corresponding derivatives. Write $P_{n}^{*}(x)=\|x\| P_{n}\left(\|x\|^{-1} x\right)$ for $x$ $\in E^{d} \backslash\{0\}$; then $H$ and its first and second derivatives are also approximated, uniformly for $\frac{1}{2} \leq\|x\| \leq 2$, by the functions $P_{n}^{*}$ and their derivatives. It follows that $\eta\left(P_{n}^{*}\right) \rightarrow \eta(H)$ for $n \rightarrow \infty$, hence $\eta\left(P_{n}^{*}\right)>0$ for sufficiently large $n$. Thus, by (3.11), almost all functions $P_{n}^{*}$ are support functions. Now $P_{n}$ is a sum of homogeneous polynomials, and every homogeneous polynomial $P$ can be written in the form

$$
P(x)=Q_{0}(x)+\|x\|^{2} Q_{1}(x)+\|x\|^{4} Q_{2}(x)+\cdots+\|x\|^{2 k} Q_{k}(x),
$$

where $Q_{0}, Q_{1}, \ldots, Q_{k}$ are harmonic homogeneous polynomials (Coifman-Weiss [7, p. 138]). Thus the restriction to $S^{d-1}$ of the function $P_{n}^{*}$, which coincides with the restriction of $P_{n}$, is an element of $\mathfrak{S}^{d}$. The proof of (3.14) is completed by the familiar remark that the set of twice continuously differentiable support functions $H$ satisfying $\eta(H)>0$ is dense in $\overbrace{}^{d}$.

4. Description of endomorphisms. In this secion we start an investigation of the endomorphisms of $\Re^{d}$. The procedure will be similar to that in [22]. We make use of spherical harmonics, which seem to be the natural tool in exploiting the rotation equivariance expressed by (1.3). The results obtained here form the basis for the proofs of the theorems stated in the introduction.

Let us first see how the well-known fact (3.6) leads to another way of constructing endomorphisms of $\Omega^{d}$. Choose a natural number $r$ and $r$ real numbers $\gamma_{0}, \gamma_{2}, \gamma_{3}, \ldots, \gamma_{r}$, and define a map $\Phi^{*}: C\left(S^{d-1}\right) \rightarrow C\left(S^{d-1}\right)$ by

$$
\Phi^{*} F=\sum_{m=0}^{r} \gamma_{m} \Pi_{m} F, \quad F \in C\left(S^{d-1}\right),
$$

with $\gamma_{1}=1$. Clearly, $\Phi^{*}$ is linear and continuous, and it satisfies

$$
\Phi^{*} g=g \Phi^{*} \quad \text { for } g \in S O(d) .
$$


Let us assume for the moment that $\Phi^{*}$ maps the cone $\widetilde{5}^{d}$ of support functions into itself. Then we can define a map $\Phi: \Re^{d} \rightarrow \Omega^{d}$ by letting $\Phi K$ be the convex body with support function $\Phi^{*} H_{K}$. Evidently, $\Phi$ has properties (1.1) and (1.2). Since $H_{g K}=g H_{K}$ for $g \in S O(d)$ and $K \in \Omega^{d}$, (4.2) implies $\Phi g K=g \Phi K$ for $g \in S O(d)$ and $K \in \Re^{d}$. Since, for $t \in E^{d}$, the support function of the convex body $\{t\}$ is given by $H_{\{t\}}(u)=t \cdot u$ for $u \in S^{d-1}$ and hence is an element of $\mathfrak{S}_{1}^{d}$, we have $\Phi\{t\}=\{t\}$ because of $\gamma_{1}=1$. This shows that $\Phi(K+t)=\Phi K+\Phi\{t\}$ $=\Phi K+t$. Thus $\Phi$ has property (1.3).

It remains to show that the constants $\gamma_{0}, \gamma_{2}, \gamma_{3}, \ldots, \gamma_{r}$ can in fact be chosen in many nontrivial ways such that the map $\Phi^{*}$ defined by (4.1) maps the cone $\Im^{d}$ into itself. Let us denote by $\mathbb{E}_{0}^{d}$ the set of those functions $F \in \mathbb{\mho}^{d}$ which satisfy $\Pi_{0} F=1$ and $\Pi_{1} F=0$ (thus, by (3.9) and (3.10), $\bigodot_{0}^{d}$ contains exactly the support functions of the convex bodies of mean width 2 whose Steiner point coincides with the origin). Now choose an orthonormal basis $Y_{m 1}, \ldots, Y_{m N_{m}}$ in each of the spaces $\mathfrak{E}_{m}^{d}, m=2,3, \ldots$ Put $N=N_{2}+\cdots+N_{r}$, and let $\mathbf{R}^{N}$ be the space of real $N$-tuples. Define two subsets $A, C \subset \mathbf{R}^{N}$ in the following way: $A$ is the set of all $N$-tuples $\left(a_{21}, \ldots, a_{2 N_{2}}, a_{31}, \ldots, a_{3 N_{3}}, \ldots, a_{r 1}, \ldots, a_{r N_{r}}\right) \in \mathbf{R}^{N}$ with the property that there exists a support function $F \in \mathfrak{S}_{0}^{d}$ such that

$$
\left\langle F, Y_{m j}\right\rangle=a_{m j} \text { for } j=1, \ldots, N_{m}, m=2,3, \ldots, r,
$$

or, in other words,

$$
\Pi_{m} F=\sum_{j=1}^{N_{m}} a_{m j} Y_{m j} \text { for } m=2,3, \ldots, r .
$$

$C$ is the set of all $N$-tuples $\left(c_{21}, \ldots, c_{2 N_{2}}, c_{31}, \ldots, c_{3 N_{3}}, \ldots, c_{r 1}, \ldots, c_{r N_{r}}\right) \in \mathbf{R}^{N}$ with the property that the function

$$
1+\sum_{m=2}^{r} \sum_{j=1}^{N_{m}} c_{m j} Y_{m j}
$$

belongs to $\mho_{0}^{d}$. We observe that $A$ is bounded: If (4.3) holds with $F \in \mathcal{F}_{0}^{d}$, then by Bessel's inequality,

$$
\sum_{m=2}^{r} \sum_{j=1}^{N_{m}}\left|a_{m j}\right|^{2} \leq \int_{S^{d-1}}|F|^{2} d \omega .
$$

Now $F$ is the support function of some convex body $K$ of mean width 2 and with its Steiner point in the origin. Since every convex body of this kind is contained in some sufficiently large ball independent of $K$, there is some bound for $|F|$ independent of $F$. The assertion follows. Next we remark that $C$ contains a neighborhood of the origin in $\mathbf{R}^{N}$. Since the functions $Y_{m j}$ are twice continuously differentiable, it follows from (3.13) that the function defined by (4.4) belongs to $\mathbb{S}_{0}^{d}$ if $c_{21}^{2}+\cdots+c_{r N_{r}}^{2}$ is smaller than some fixed positive constant. 
It is now clear that we can choose balls $B_{\rho}^{N}, B_{o}^{N}$ in $\mathbf{R}^{N}$ centered at the origin and with (positive) radii $\rho, \sigma$, respectively, such that $B_{\rho}^{N} \subset C$ and $A \subset B_{\sigma}^{N}$. Let $\lambda \in \mathbf{R}$ be a number with $0<\lambda \sigma<\rho$, and choose the real numbers $\gamma_{2}, \gamma_{3}, \ldots$, $\gamma_{r}$ such that $\left|\gamma_{m}\right|<\lambda(m=2, \ldots, r)$. Now define $\Phi^{*}$ by (4.1) with $\gamma_{0}=\gamma_{1}=1$. Then if $F \in \mathbb{\complement}_{0}^{d}$ and $a_{m j}$ is defined by (4.3), we have $\left(a_{2 !}, \ldots, a_{r N_{r}}\right) \in A \subset B_{\sigma}^{N}$, and

$$
\Phi^{*} F=1+\sum_{m=2}^{r} \gamma_{m} \sum_{j=1}^{N_{m}} a_{m j} Y_{m j}
$$

From $\sum_{m=2}^{r} \sum_{j=1}^{N_{m}}\left(\gamma_{m} a_{m j}\right)^{2}<\lambda^{2} \sigma^{2}<\rho^{2}$ it follows that $\left(\gamma_{2} a_{21}, \ldots, \gamma_{r} a_{r N_{r}}\right) \in B_{\rho}^{N}$ $\subset C$, which means that $\Phi^{*} F \in \widetilde{\mho}_{0}^{d}$. Thus $\Phi^{*} F$ is a support function whenever $F$ is the support function of a convex body with mean width 2 and Steiner point in the origin. But then it follows that $\Phi^{*}$ maps $\varpi^{d}$ into $\widetilde{\mho}^{d}$. Hence the map $\Phi: \Re^{d} \rightarrow \Re^{d}$ defined by $H_{\Phi K}=\Phi^{*} H_{K}$ is an endomorphism of $\Re^{d}$.

The examples just constructed exhibit, to a certain extent, the general character of endomorphisms. Let $\Phi$ be an endomorphism of $\Re^{d}$. The map $\Phi$ gives rise to an equivariant linear operator on a suitable vector space: If $F$ is an element of $\mathfrak{Q}^{d}$, the space of generalized trigonometric polynomials on $S^{d-1}$, then the function $F$ is twice continuously differentiable; hence by (3.12) there are convex bodies $K, L \in \Re^{d}$ such that $F=H_{K}-H_{L}$. We can define $\Phi^{*} F=H_{\Phi K}-H_{\Phi L}$, since this function depends only on $F$ and not on the special representation of $F$ as a difference of support functions. It follows from the properties of $\Phi$ that the map $\Phi^{*}$ thus defined is a linear operator from $\mathfrak{S}^{d}$ into $C\left(S^{d-1}\right)$ which commutes with the action of the rotation group. We remark that for a continuous linear operator from $C\left(S^{d-1}\right)$ into itself which commutes with rotations, there exists an explicit representation as a kind of convolution operator closely related to formula (2.4); see Dunkl $[10, \S 8]$. However, this does not help us in our case since the operator $\Phi^{*}$ is only defined on a proper subspace of $C\left(S^{d-1}\right)$ and is not known to be continuous there. It is known, however, that a linear operator, defined on generalized trigonometric polynomials (with values in a suitable function space) which commutes with the translation operators induced by the group action, must be of a special nature (which in our case is expressed by (4.5) and (4.6) below), whence such an operator is usually called a "multiplier operator" (see, e.g., Coifman-Weiss [8, pp. 55-56]). We include an elementary proof for the sake of completeness.

Let $m \in\{0,1,2, \ldots\}$, and let $\Phi_{m}^{*}$ be the restriction of $\Phi^{*}$ to the space $\mathfrak{Y}_{m}^{d}$ of spherical harmonics of degree $m$. For $k \in\{0,1,2, \ldots\}$ the map $\Pi_{k} \Phi_{m}^{*}$ is a linear operator from $\mathfrak{S}_{m}^{d}$ to $\mathfrak{S}_{k}^{d}$ which commutes with rotations. Since $\mathfrak{S}_{m}^{d}$ and $\mathfrak{S}_{k}^{d}$ are irreducible, it follows from the lemma of Schur (see, e.g., Boerner [4, p. 21]) that $\Pi_{k} \Phi_{m}^{*}$ is either zero or an isomorphism. The assumption $\Pi_{k} \Phi_{m}^{*} \neq 0$ implies $k=m$, since $\operatorname{dim} \mathfrak{E}_{m}^{d} \neq \operatorname{dim} \mathfrak{S}_{k}^{d}$ if $k \neq m$ and $d \geq 3$; for $d=2$ the equality $k=m$ is an elementary consequence of the rotation equivariance of $\Pi_{k} \Phi_{m}^{*}$. 
Equally easy to see is the fact that, for $d=2$,

$$
\Pi_{m} \Phi_{m}^{*} F=\gamma_{m} g_{m} F \text { for } F \in \mathscr{S}_{m}^{2},
$$

where $\gamma_{m}$ is a real number and $g_{m} \in S O(2)$ is a rotation. Now let $d \geq 3$. For an arbitrary point $p \in S^{d-1}$ let $G_{p}$ denote the subgroup of $S O(d)$ which leaves $p$ fixed. The function $Z_{p}$ defined by $Z_{p}(u)=C_{m}^{v}(u \cdot p)$ for $u \in S^{d-1}$ is invariant under $G_{p}$, and up to a constant factor it is the only element of $\mathfrak{\mathcal { O }}_{m}^{d}$ with this property. For $g \in G_{p}$ we have

$$
g \Pi_{m} \Phi_{m}^{*} Z_{p}=\Pi_{m} \Phi_{m}^{*} g Z_{p}=\Pi_{m} \Phi_{m}^{*} Z_{p},
$$

hence $\Pi_{m} \Phi_{m}^{*} Z_{p}=\gamma_{m}(p) Z_{p}$ with a real constant $\gamma_{m}(p)$. Replacing $p$ by $h p$ with $h \in S O(d)$ and observing $Z_{h p}=h Z_{p}$, we see that $\gamma_{m}(h p)=\gamma_{m}(p)$; hence, by the transitivity of $S O(d)$ on $S^{d-1}, \gamma_{m}(p)$ does not depend on $p$. Since the functions $Z_{p}\left(p \in S^{d-1}\right)$ linearly span $\mathfrak{Q}_{m}^{d}$, we conclude that $\Pi_{m} \Phi_{m}^{*} F=\gamma_{m} F$ for $F \in \mathfrak{\mathcal { Q }}_{m}^{d}$. We have proved that

$$
\begin{aligned}
\Pi_{m} \Phi^{*} F & =\gamma_{m} F, & & \text { if } F \in \mathscr{\bigotimes}_{m}^{d}, \\
& =0, & & \text { if } F \in \mathfrak{Q}_{k}^{d} \text { and } k \neq m ;
\end{aligned}
$$

hence, by linearity,

$$
\Pi_{m} \Phi^{*} F=\gamma_{m} \Pi_{m} F
$$

for $F \in \mathscr{S}^{d}$, provided that $d \geq 3$. For $d=2$ it is clear that (4.5) has to be replaced by

$$
\Pi_{m} \Phi^{*} F=\gamma_{m} g_{m} \Pi_{m} F
$$

for $F \in \mathscr{Q}^{2}$, where $g_{m} \in S O(2)$.

It remains to show that equation (4.5), respectively (4.6), carries over to an arbitrary support function $F$. This follows from the continuity of $\Phi$, which implies the continuity of $\Phi^{*}$ on the cone $\mho^{d}$ of support functions, and from Lemma (3.14).

We resume the result, together with some supplements, as a lemma.

(4.7) Lemma. Let $\Phi$ be an endomorphism of $\Re^{d}$. Then there is a sequence $\left(\gamma_{0}, \gamma_{1}, \gamma_{2}, \ldots\right)$ of real numbers, and an additional sequence $\left(g_{0}, g_{1}, g_{2}, \ldots\right)$ of rotations $g_{i} \in S O(2)$ if $d=2$, such that

$$
\Pi_{m} H_{\Phi K}=\gamma_{m} \Pi_{m} H_{K} \quad \text { for each } K \in \Omega^{d}
$$

if $d \geq 3$, and

$$
\Pi_{m} H_{\Phi K}=\gamma_{m} g_{m} \Pi_{m} H_{K} \text { for each } K \in \AA^{2}
$$

if $d=2(m=0,1,2, \ldots)$; here $\gamma_{1}=1$, and $g_{0}, g_{1}$ are equal to the identity. The 
endomorphism $\Phi$ is uniquely determined by the sequence $\left(\gamma_{0}, \gamma_{1}, \gamma_{2}, \ldots\right)$, together with $\left(g_{0}, g_{1}, g_{2}, \ldots\right)$ if $d=2$.

That $\Phi$ is uniquely determined by its associated sequence (respectively sequences, if $d=2$ ), follows from the completeness of the system of spherical harmonics. That $g_{0}$ may be chosen as the identity is trivial, since $\mathfrak{S}_{0}^{d}$ contains only constant functions. The translation equivariance of the endomorphism $\Phi$ implies $\Phi\{p\}=\{p\}$ for $p \in E^{d}$; since $H_{\{p\}} \in \mathfrak{Q}_{1}^{d}$, this leads to $\gamma_{1}=1$ and $g_{1}=$ identity. We remark that (3.9) and (3.10) now exhibit the following behaviour of the mean width and the Steiner point under an endomorphism $\Phi$ :

$$
\begin{aligned}
& \beta(\Phi K)=\gamma_{0} \beta(K), \\
& s(\Phi K)=s(K),
\end{aligned}
$$

Of course, the fact that $\Phi^{*} F$ is a support function whenever $F$ is imposes severe restrictions upon the sequences $\left(\gamma_{0}, \gamma_{1}, \gamma_{2}, \ldots\right)$ thus obtainable. It seems difficult to find necessary and sufficient conditions for a sequence $\left(\gamma_{i}\right)$ to come from a map $\Phi^{*}$ which maps the cone $\overleftarrow{C}^{d}$ of support functions into itself; however, the information already available suffices to prove Theorem (1.7). We note one obvious restriction for the sequence $\left(\gamma_{i}\right)$, which will be needed later in the proof of the other theoems.

(4.12) Lemma. If $\Phi$ is an endomorphism of $\AA^{d}$ and if $\left(\gamma_{i}\right)$ denotes the sequence of real numbers associated with $\Phi$ according to Lemma (4.7), then $\left|\gamma_{m}\right| \leq \gamma_{0}$ for $m=2,3,4, \ldots$.

Proof. Let us first assume that $\gamma_{0}=0$. By (4.10) this implies $\beta(\Phi K)=0$ for $K \in \Omega^{d}$; hence $\Phi K$ contains only one point. But then $H_{\Phi K} \in \mathfrak{S}_{1}^{d}$, so that $\Pi_{m} H_{\Phi K}=0$ for $m \neq 1$. From Lemma (4.7) we deduce that $\gamma_{m}=0$, since $\Pi_{m} H_{K} \neq 0$ for suitable $K$, if $m \neq 1$ is given.

Now assume $\gamma_{0} \neq 0$; hence $\gamma_{0}>0$ by (4.10). Let $m \in\{2,3, \ldots\}$, and put

$$
J_{m}=\left\{\alpha \in \mathbf{R} \mid u \mapsto 1+\alpha C_{m}^{\gamma}(u \cdot e) \text { is a support function }\right\},
$$

where $e \in S^{d-1}$ is a fixed vector. It follows from (3.13) that $J_{m}$ contains a neighborhood of 0 , and it is clear that $J_{m}$ is a compact subset of $\boldsymbol{R}$ (if $H_{K}(u)=1+\alpha C_{m}^{\nu}(u \cdot e)$ for some $K \in \Omega^{d}$, then $s(K)=0$ and hence $H_{K} \geq 0$; as $C_{m}^{\nu}(u \cdot e)$ attains values of either sign, $J_{m}$ must be bounded). Choose $\alpha \in J_{m}$, and let $K \in \Omega^{d}$ be the convex body which satisfies $H_{K}(u)=1+\alpha C_{m}^{v}(u \cdot e)$ for $u \in S^{d-1}$. By Lemma (4.7) we have

$$
H_{\Phi K}(u)=\gamma_{0}+\alpha \gamma_{m} C_{m}^{v}\left(\left[g_{m}^{-1} u\right] \cdot e\right), \quad u \in S^{d-1}
$$

(with $g_{m}=$ identity if $d \geq 3$ ). Since the function $\gamma_{0}^{-1} H_{\Phi K}$ is a support function, we have $\alpha \gamma_{0}^{-1} \gamma_{m} \in J_{m}$. As this must be true for arbitrary $\alpha \in J_{m}$, the assertion $\left|\gamma_{0}^{-1} \gamma_{m}\right| \leq 1$ follows. 
As an important consequence of (4.12) we have the following result. By $W_{k}(K)$ we denote the $k$ th quermassintegral of $K(k=0,1, \ldots, d-1)$.

(4.14) Lemma. If $\Phi$ is an endomorphism of $\AA^{d}$ and if $\left(\gamma_{i}\right)$ denotes the associated sequence, then

$$
W_{d-2}(\Phi K) \geq \gamma_{0}^{2} W_{d-2}(K) \text { for } K \in \Re^{d} .
$$

The equality sign holds for all $K \in \Omega^{d}$ if and only if $\left|\gamma_{m}\right|=\gamma_{0}$ for $m=2,3$, $4, \cdots$

Proof. The functional $W_{d-2}$ can be expressed by

$$
W_{d-2}(K)=\frac{1}{d}\left\{a_{0}^{2}(K)-\frac{1}{d-1} \sum_{m=2}^{\infty}(m-1)(m+d-1) a_{m}^{2}(K)\right\},
$$

with $a_{m}^{2}(K)=\int_{S^{d-1}}\left|\Pi_{m} H_{K}\right|^{2} d \omega, m=0,1,2, \ldots$, for $K \in \Re^{d}$. For $d=3$ this formula may be read off from Blaschke [3, pp. 108-110]; for the general case it was proved by Kubota [17, p. 92]. From (4.15), Lemma (4.7), and Lemma (4.12) the inequality of Lemma (4.14) follows. The equality sign holds if $\left|\gamma_{m}\right|=\gamma_{0}$ for $m=2,3,4, \ldots$, and only in this case, since for each $m \in\{2,3,4, \ldots\}$ there exists a convex body $K \in \Re^{d}$ for which $a_{m}(K) \neq 0$.

Lemma (4.14) will mainly be utilized in connection with the following lemma, which expresses a special case of well-known properties of mixed volumes (Bonnesen-Fenchel [5, p. 41]).

(4.16) Lemma. Let $K \in \Omega^{d}$ and $k \in\{0,1, \ldots, d-1\}$. Then $W_{k}(K)=0$ if and only if the dimension of $K$ is at most $d-k-1$.

5. Proof of Theorem (1.7). Let us first suppose that $L \in \Re^{d}$ is a convex body whose support function $H_{L}$ has the property that

$$
\Pi_{m} H_{L} \neq 0 \text { for } m=2,3, \ldots .
$$

For the moment we shall say that such a body is universal in $E^{d}$. We remark that the inequality $\Pi_{0} H_{L} \neq 0$ is necessarily satisfied, since $L$ contains more than one point if (5.1) holds and hence has positive mean width.

Let $\Phi$ be an endomorphism of $\Re^{d}$. For $d \geq 3$ it follows from Lemma (4.7) that $\gamma_{m} \Pi_{m} H_{L}=\Pi_{m} H_{\Phi L}$; hence $\gamma_{m}$ is known for $m=0,1,2, \ldots$ if $\Phi L$ is known (observe that $\gamma_{1}=1$ ). Thus, by Lemma (4.7), $\Phi$ is uniquely determined by its value at $L$. In case $d=2$ it follows from Lemma (4.7) that $\gamma_{m} g_{m} \Pi_{m} H_{L}$ $=\Pi_{m} H_{\Phi L}$. Again $\gamma_{m}$ is uniquely determined for $m=0,1,2, \ldots$, and $g_{m}$ is determined up to a rotation by an angle which is an integer multiple of $2 \pi / \mathrm{m}$; hence $\Phi$ is uniquely determined.

According to these observations, Theorem (1.7) will be proved when we have shown that a triangle with at least one irrational angle is universal in $E^{d}$. Perhaps the reader may wonder why we have picked out just a triangle in the formulation 
of the theorem. The reason is, firstly, that a triangle is a convex body which is easy to describe, and secondly, that we do not know of any other definite convex body for which the property of being universal could be verified without cumbersome computations. Furthermore, the existence of a triangle which is universal in $E^{d}$ can be utilized in the proof of (1.8).

Let $T$ be a triangle with an irrational angle. First we consider $T$ as a subset of $E^{2}$. Suppose that $T$ were not universal in $E^{2}$. Then there exists a number $m \in\{2,3, \ldots\}$ such that $\Pi_{m} H_{T}=0$. Let $K \subset E^{2}$ be the convex body with support function $H_{K}=1+\alpha Y_{m}$, where $Y_{m} \not \equiv 0$ is a spherical harmonic on $S^{1}$ (that is, a certain trigonometric function) of degree $m$, and where $\alpha \neq 0$ is chosen appropriately (according to (3.13)). Let $A\left(K_{1}, K_{2}\right)$ denote the mixed surface area of the convex bodies $K_{1}, K_{2} \in \Omega^{2}$. From the assumptions on $T$ and $K$ it follows that $A(T, g K)$ is constant for all $g \in S O(2)$ (Bonnesen-Fenchel [5, p. 139]). If $u_{1}, u_{2}, u_{3} \in S^{1}$ denote the exterior unit normal vectors of the triangle $T$ and $\sigma_{1}, \sigma_{2}, \sigma_{3}$ are the lengths of the corresponding sides, we have (Bonnesen-Fenchel $[5,(3)$, p. 41])

$$
A(T, g K)=\frac{1}{2} \sum_{i=1}^{3} H_{g K}\left(u_{i}\right) \sigma_{i}=\frac{1}{2} \sum_{i=1}^{3} \sigma_{i} H_{K}\left(g^{-1} u_{i}\right) .
$$

As $A(T, g K)$ is constant, this gives

$$
\sum_{i=1}^{3} \sigma_{i}\left[H_{K}\left(g u_{i}\right)-R\right]=0 \text { for all } g \in S O(2),
$$

where $R>0$ is a suitable constant. In view of the relation $\sum_{i=1}^{3} \sigma_{i} u_{i}=0$ this means that $K$ is a rotor of a triangle similar to $T$ and with incircle radius $R$ (see, e.g., Schneider [23, Hilfssatz 2.2]). But it is well known that the only triangles which admit noncircular rotors are those with only rational angles (see Schaal [21] for a short proof as well as for further references). This proves that $T$ is universal in $E^{2}$.

Now we show that a convex body which is universal in $E^{d-1}$ is also universal in $E^{d}(d \geq 3)$. An inductive argument then shows that $T$ is universal in $E^{d}$.

Let $K \subset E^{d}(d \geq 3)$ be a convex body which is at most $(d-1)$-dimensional and which, when considered as a subset of $E^{d-1}$, is universal in that space. We may assume that $K \subset E^{d-1} \subset E^{d}$. Let $e \in E^{d}$ be one of the two unit vectors orthogonal to $E^{d-1}$, and let $S^{d-2}=S^{d-1} \cap E^{d-1}$ be the unit sphere in $E^{d-1}$. Each unit vector $u \in S^{d-1}$ can uniquely be written in the form

$$
u=t e+\left(1-t^{2}\right)^{1 / 2} \bar{u}, \quad \text { where } \bar{u} \in S^{d-2},
$$

so that $t=u \cdot e$. If $h_{K}: S^{d-2} \rightarrow \mathbf{R}$ denotes the support function of $K$, the latter being considered as a body in $E^{d-1}$, the support function $H_{K}$ of $K$ with respect to $E^{d}$ is given by 


$$
H_{K}(u)=\left(1-t^{2}\right)^{1 / 2} h_{K}(\bar{u}), \quad u \in S^{d-1},
$$

where $t$ and $\bar{u}$ are defined by (5.2).

Let $m \in\{2,3, \ldots\}$. We have to show that $\Pi_{m} H_{K} \neq 0$ or, equivalently, that $\left\langle H_{K}, F\right\rangle \neq 0$ for at least one spherical harmonic $F \in \mathfrak{S}_{m}^{d}$. Since $K$ is universal in $E^{d-1}$, there exists a spherical harmonic $f \in \mathfrak{S}_{m}^{d-1}$ such that $\left\langle h_{K}, f\right\rangle \neq 0$, where now $\langle$, $\rangle$ denotes the usual inner product for functions on $S^{d-2}$. Now the function $F$ defined by

$$
F(u)=\left(1-t^{2}\right)^{m / 2} f(\bar{u}), \quad u \in S^{d-1},
$$

is a spherical harmonic of degree $m$ on $S^{d-1}$, since the function $t \mapsto\left(1-t^{2}\right)^{m / 2}$ is an associated Legendre function of degree $m$, order $m$, and dimension $d$ (compare Müller [19, pp. 22-25]). If $\bar{\omega}$ denotes $(d-2)$-dimensional Lebesgue measure on $S^{d-2}$, we get (using Müller $[19$, p. 1])

$$
\begin{aligned}
\left\langle H_{K}, F\right\rangle & =\int_{S^{d-1}} H_{K}(u) F(u) d \omega(u) \\
& =\int_{S^{d-2}} \int_{-1}^{1}\left(1-t^{2}\right)^{1 / 2} h_{K}(\bar{u})\left(1-t^{2}\right)^{m / 2} f(\bar{u})\left(1-t^{2}\right)^{v-1 / 2} d t d \bar{\omega} \\
& =\int_{-1}^{1}\left(1-t^{2}\right)^{v+m / 2} d t\left\langle h_{K}, f\right\rangle \neq 0 .
\end{aligned}
$$

The proof of Theorem (1.7) is now complete.

6. Proof of Theorem (1.8) and its corollaries. Let $\Phi$ be an endomorphism of $\Re^{d}$. First we assume that for some convex body $L \in \Omega^{d}$, which is at least onedimensional, the image $\Phi L$ contains only one point. Then $\beta(L)>0$ and $\beta(\Phi L)=0$, which implies $\gamma_{0}=0$ and hence $\beta(\Phi K)=0$ for each $K \in \Re^{d}$ by (4.10). Thus $\Phi K$ contains only one point, so that $\Phi K=\{s(\Phi K)\}=\{s(K)\}$ by (4.11). This proves part (a) of Theorem (1.8).

Now let us assume that, for some $L \in \Omega^{d}$, the image $\Phi L$ is a (nondegenerate) segment. Then $W_{d-2}(\Phi L)=0$ by (4.16), and $\gamma_{0} \neq 0$ by (4.10); hence (4.14) implies $W_{d-2}(L)=0$ which, again by (4.16), shows that $L$ is a segment. Without loss of generality (compare (4.11)) we may assume that both $L$ and $\Phi L$ have its centre at the origin of $E^{d}$. Thus there exists a nonnegative number $\lambda$ and a rotation $g \in S O(d)$ such that

$$
\Phi L=\lambda g L .
$$

For $d \geq 3, g$ may be chosen as the identity, since $\Phi$ commutes with rotations and since there exist rotations under which exactly those segments (with centre at the origin) remain fixed which are multiples of $L$. By (6.1), Lemma (4.7), and (3.6) we have

$$
\lambda g \Pi_{m} H_{L}=\Pi_{m} H_{\lambda g L}=\Pi_{m} H_{\Phi L}=\gamma_{m} g_{m} \Pi_{m} H_{L}
$$


for $m=0,1,2, \ldots$, where $\left(\gamma_{i}\right)$ and $\left(g_{i}\right)$ are the sequences associated with $\Phi$ according to (4.7). Here we have to put $g_{m}=$ identity if $d \geq 3$. Now (3.8) and (3.7) show that

$$
\left(\Pi_{m} H_{L}\right)(u)=|L| \beta_{m}^{v} \eta_{m}^{>} \lambda_{m}^{>} C_{m}^{\nu}(u \cdot p), \quad u \in S^{d-1},
$$

where $2|L|$ is the length of $L$ and $p$ is a unit vector parallel to $L$; furthermore, $\beta_{m}^{\prime} \eta_{m}^{\prime} \neq 0$ and

$$
\lambda_{m}^{\prime}=\int_{-1}^{1}|t| C_{m}^{\gamma}(t)\left(1-t^{2}\right)^{p-1 / 2} d t
$$

With the aid of (3.3) one computes that $\lambda_{m}^{\nu} \neq 0$ for even $m$. Hence from (6.2) we deduce, for even $m$, that $\gamma_{m}=\lambda$ and that, in the case $d=2, g^{-1} g_{m}$ is a rotation by an angle which is an integer multiple of $2 \pi / m$. Thus we have

$$
\gamma_{m} g_{m} F=\lambda g F \text { for } F \in \mathfrak{S}_{m}^{d}, m=0,2,4, \ldots
$$

Now let us define an endomorphism $\Psi$ of $\AA^{d}$ by

$$
\Psi K=\lambda_{1} g[K-s(K)]+\lambda_{2} g[-K+s(K)]+s(K) \text { for } K \in \Re^{d},
$$

where $0 \leq \lambda_{1} \leq \lambda$ and $\lambda_{1}+\lambda_{2}=\lambda$. A definite choice for $\lambda_{1}$ will be made later. Let $K \in \Omega^{d}$ be a convex body which is centrally symmetric with respect to the origin (so that $s(K)=0$ ). Then $\Pi_{m} H_{K}=0$ for odd $m$, since $H_{K}$ is an even function and each element of $\mathfrak{S}_{m}^{d}$ is an odd function; thus $\Pi_{m} H_{\Phi K}=0$ by (4.8) or (4.9). As $\Psi K$ is centrally symmetric with respect to the origin, we have $\Pi_{m} H_{\Psi K}=0$; thus

$$
\Pi_{m} H_{\Phi K}=\Pi_{m} H_{\Psi K} \text { for } m=1,3,5, \ldots
$$

Let $m$ be even. Directly from (6.4) and $\lambda_{1}+\lambda_{2}=\lambda$ we get $\Pi_{m} H_{\Psi K}$ $=\lambda g \Pi_{m} H_{K}$. From (4.7) and (6.3) we deduce

$$
\Pi_{m} H_{\Phi K}=\gamma_{m} g_{m} \Pi_{m} H_{K}=\lambda g \Pi_{m} H_{K} .
$$

Thus we have shown that

$$
\Pi_{m} H_{\Phi K}=\Pi_{m} H_{\Psi K} \text { for } m=0,1,2, \ldots
$$

Now the completeness of the system of spherical harmonics implies $H_{\Phi K}=H_{\Psi K}$. As $\Phi$ and $\Psi$ both commute with translations, it follows that

$$
\Phi K=\Psi K \text { for each centrally symmetric } K \in \Re^{d} \text {. }
$$

To see what $\Phi$ does with a not centrally symmetric convex body, let $T \in \Re^{d}$ be a triangle with at least one irrational angle. Then $T+(-T)$ is centrally symmetric with respect to the origin; hence (6.5) yields 


$$
\Phi T+\Phi(-T)=\Phi(T+(-T))=\Psi(T+(-T))=\lambda g(T+(-T)) .
$$

From Lemma (4.7) and the fact that (3.6) holds also for improper rotations $g$, it is clear that $\Phi(-K)=-\Phi K$ for $K \in \Omega^{d}$, even if reflection in the origin does not belong to $S O(d)$ (i.e. for odd $d$ ). Hence $\Phi T+(-\Phi T)=\lambda g T+(-\lambda g T)$. An elementary discussion of the possible decompositions of the centrally symmetric hexagon $\lambda g T+(-\lambda g T)$ into a sum of two convex bodies shows that the above equality is possible only if $\Phi T$ is a translate of the body $\lambda_{1} g T+\left(-\lambda_{2} g T\right)$, where $\lambda_{1}, \lambda_{2}$ are real numbers, $0 \leq \lambda_{i} \leq \lambda$, and $\lambda_{1}+\lambda_{2}=\lambda$. Then (4.11) implies that

$$
\Phi T=\lambda_{1} g[T-s(T)]+\lambda_{2} g[-T+s(T)]+s(T) .
$$

Using these numbers $\lambda_{1}, \lambda_{2}$ in definition (6.4) of the endomorphism $\Psi$, we arrive at $\Phi T=\Psi T$. Theorem (1.7) now shows that $\Phi=\Psi$, which proves part (b) of Theorem (1.8).

Theorem (1.4) is an easy corollary of (1.8). In fact, let $\Phi$ be an endomorphism of $\Re^{d}$ which satisfies $\Phi a K=a \Phi K$ for each affine map $a: E^{d} \rightarrow E^{d}$. Let $S$ be a segment with centre at the origin of $E^{d}$, and let $L$ be the line which carries $S$. Since there are affine transformations of $E^{d}$ which leave $S$ fixed but do not leave fixed any element of $\Re^{d}$ which is not a subset of $L$, we see that $\Phi S$ must be contained in $L$ and hence is either one-pointed or a segment. In the first case, (1.8) (a) implies $\Phi K=\{s(K)\}$ for $K \in \Re^{d}$, a contradiction since the Steiner point is not affine-equivariant. Hence $\Phi S$ is a segment, and now (1.8)(b) shows that

$$
\Phi K=\lambda g[K-s(K)]+\mu g[-K+s(K)]+s(K) \text { for } K \in \Re^{d}
$$

with suitable $\lambda, \mu, g$. If $g$ is not the identity or reflection in the origin, there exists an affine transformation $a$ of $E^{d}$ and a centrally symmetric convex body $K \in \Omega^{d}$ such that $g a K \neq a g K$, which contradicts $\Phi a K=a \Phi K$. Hence we may assume that $g$ is the identity. Since $s(a K) \neq a s(K)$ for suitable $K \in \Re^{d}$ and some affine transformation $a$, it follows that $\lambda=1+\mu$. Thus (1.4) is proved.

Corollary (1.9) follows immediately from (1.8)(b) and the observation that either $\lambda$ or $\mu$ must be equal to zero, since otherwise a triangle, which is indecomposable, cannot be attained as an image. Corollary (1.9) trivially implies Corollary (1.10). Corollary (1.12) follows at once from (1.8)(b): If $d \geq 3$ and if the image of a segment is not a segment, it cannot be a polytope, since it must have rotational symmetry. Hence the image of a $d$-dimensional zonotope (sum of segments) cannot be a polytope, which contradicts the assumption.

In order to prove Corollary (1.13) let $k \in\{0,1, \ldots, d-2\}$, and let $\Phi$ be an endomorphism of $\Re^{d}$ which satisfies

$$
W_{k}(\Phi K)=W_{k}(K) \text { for } K \in \Re^{d} .
$$

Let $S \in E^{d}$ be a segment with centre at the origin. We want to show that $\Phi S$, too, is a segment. Clearly $\Phi S$ contains more than one point, since otherwise 
(1.8)(a) and (6.6) would lead to a contradiction. By (4.16), $W_{k}(S)=0$; hence (6.6) implies $W_{k}(\Phi S)=0$. If $d=2,(4.16)$ shows that $\Phi S$ is a segment. Now let $d \geq 3$. If $G$ denotes the subgroup of $S O(d)$ which leaves $S$ fixed, we have $\{g x \mid g \in G\} \subset \Phi S$ for $x \in \Phi S$; hence $\Phi S$ either is a segment or has dimension at least $d-1$. The latter case is only possible if $k=0$ (since otherwise $\left.W_{k}(\Phi S) \neq 0=W_{k}(S)\right)$. In this case, let $g \in S O(d)$ be a rotation such that the segments $S$ and $g S$ are not parallel. Then $W_{0}(S+g S)=0$; hence $W_{0}(\Phi(S$ $+g S))=0$, whereas the body $\Phi(S+g S)=\Phi S+g \Phi S$ has dimension $d$, a contradiction. Hence $\Phi S$ is a segment. Now it follows from (1.8)(b) that

$$
\Phi K=\lambda g[K-s(K)]+\mu g[-K+s(K)]+s(K) \text { for } K \in \Re^{d}
$$

with suitable real numbers $\lambda, \mu \geq 0$ and a rotation $g \in S O(d)$ (which is the identity if $d \geq 3$ ). Choosing $K=B$ (unit ball), we see that $\lambda+\mu=1$. Let $K \in \AA^{d}$ be a convex body with $s(K)=0$ which is not centrally symmetric. An application of the generalized Brunn-Minkowski theorem (see, e.g., Busemann [6, p. 49]) gives

$$
\begin{aligned}
W_{k}^{1 /(d-k)}(\Phi K) & =W_{k}^{1 /(d-k)}(\lambda g K+(1-\lambda) g(-K)) \\
& \geq \lambda W_{k}^{1 /(d-k)}(g K)+(1-\lambda) W_{k}^{1 /(d-k)}(-g K) \\
& =W_{k}^{1 /(d-k)}(K) .
\end{aligned}
$$

By (6.6) the equality sign must hold, which implies that either $g K$ and $-g K$ are homothetic, or $\lambda(1-\lambda)=0$. As $K$ is not centrally symmetric, $\lambda=0$ or $\lambda=1$ are the only possible cases. Thus $\Phi$ is of the form asserted in (1.13).

7. Counterexamples. Concluding we wish to emphasize the role that the continuity of the endomorphisms considered plays in the foregoing investigation. Already the example, described in $\S 1$, of a map $\Phi: \Omega^{2} \rightarrow \Omega^{2}$ which is additive in the sense of Minkowski, equivariant with respect to affine transformations, but not continuous, shows that the essential results proved above, i.e. (4.7), (1.7), (1.8), are not true without the assumption of continuity. We can also give various examples of additive, motion-equivariant maps $\Phi: \Omega^{d} \rightarrow \Re^{d}$ which are not continuous; here $d \geq 2$ is arbitrary. For instance,

$$
\Phi: K \mapsto K-s(K)+s^{\prime}(K), \quad K \in \Re^{d},
$$

is such a map, if $s^{\prime}: \AA^{d} \rightarrow E^{d}$ is a noncontinuous "Steiner point", as constructed by Sallee [20]. However, this example is perhaps not very instructive, since $\Phi K$ is always a translate of $K$, and only the translation vector $s^{\prime}-s$ is responsible for the noncontinuity. Examples of a different nature are obtained as follows.

The first surface area measure $\mu_{1}(K ; \cdot)$ of the convex body $K$ (Fenchel-Jessen [12]) is a nonnegative Borel measure on $S^{d-1}$, which depends linearly on $K$, i.e. satisfies 


$$
\mu_{1}\left(\lambda_{1} K_{1}+\lambda_{2} K_{2} ; \cdot\right)=\lambda_{1} \mu_{1}\left(K_{1} ; \cdot\right)+\lambda_{2} \mu_{1}\left(K_{2} ; \cdot\right)
$$

for $K_{1}, K_{2} \in \Omega^{d}$ and nonnegative $\lambda_{1}, \lambda_{2}$. If $\mu_{1}^{s}(K ; \cdot)$ denotes the singular part (with respect to Borel-Lebesgue measure on $S^{d-1}$ ) of $\mu_{1}(K ; \cdot)$, then it follows from the uniqueness of the Lebesgue decomposition of a measure into its singular and its absolutely continuous part that $\mu_{1}^{s}(K ; \cdot)$, too, depends linearly on $K$. By the way, this yields another example of a noncontinuous "Steiner point", namely

$$
s^{\prime}(K)=\int_{S^{d-1}} u d \mu_{1}^{s}(K ; u)+s(K), \quad K \in \Re^{d} .
$$

It is easy to see that $s^{\prime}(K) \neq s(K)$ for suitable $K \in \Re^{d}$. On the other hand, we remark that $s^{\prime}(K)=s(K)$ if $K$ is either a smooth body or a polytope.

Now define, for instance,

$$
H_{K}^{*}(u)=\int_{S^{d-l}}|u \cdot v| d \mu_{1}^{s}(K ; v)+s(K) \cdot u, \quad u \in E^{d},
$$

for $K \in \Omega^{d}$. Then $H_{K}^{*}$ is a support function, since $u \mapsto|u \cdot v|$ is a support function, and $\mu_{1}^{s} \geq 0$. If we define $\Phi: \Omega^{d} \rightarrow \Re^{d}$ by $H_{\Phi K}=H_{K}^{*}$, it is clear that $\Phi$ is additive and equivariant with respect to similarities. On a dense subset of $\Omega^{d}$, for instance on the set of smooth bodies, we have $\Phi K=\{s(K)\}$; however, if $K$ is a $d$-dimensional polytope, then $\Phi K$ is $d$-dimensional, which shows that $\Phi$ is not continuous.

Note added in proof. Theorem(1.4), as it stands, admits a direct, elementary proof. In fact, let $T: \Re^{d} \rightarrow \Omega^{d}$ be a map which commutes with all affine maps (including singular ones) of $E^{d}$ into itself. Then there exists a number $\mu \geq 0$ such that $T S=\mu[S-s(S)]+s(S)$ for each segment $S \in \Re^{d}$. Now let $K \in \Re^{d}$. Considering orthogonal projections of $K$ on to lines and using the fact that $T$ commutes with these projections, one gets

$$
H_{T K}(u)=H_{K}(u)+\frac{1}{2}(u-1)\left[H_{K}(u)+H_{K}(-u)\right], \quad u \in S^{d-1} .
$$

Clearly $\mu-1 \geq 0$ (otherwise the equality would lead to a contradiction if $K$ is a triangle), hence

$$
T K=K+\lambda[K+(-K)], \quad K \in \Re^{d},
$$

with $\lambda \geq 0$.

It remains to remark that a map $T: \Re^{d} \rightarrow \Re^{d}$ which is equivariant with respect to nonsingular affine transformations, necessarily also commutes with singular affine maps, if it is continuous. This shows that in (1.4) the assumption that the map in question be additive, is even superfluous. Theorem (1.4) should, therefore, be replaced by the following less trivial version. Here $\Re_{0}^{d} \subset \Omega^{d}$ denotes the set of convex bodies with interior points. 
$\left(1.4^{\prime}\right)$ Theorem. Every additive, continuous, affine-equivariant map $\Phi: \Re_{0}^{d} \rightarrow \Re^{d}$ is of the form

$$
\Phi K=K+\lambda[K+(-K)], \quad K \in \Re^{d},
$$

where $\lambda \geq 0$ is a real constant.

Contrasting the situation where $\Phi$ is defined and continuous on all of $\Re^{d}$, the assumption of additivity is now essential. This is shown by the examples $\Phi K=\{$ centroid of $K\}$, or $\Phi K=$ Loewner ellipsoid of $K$. It also appears that for geometrically interesting affine-equivariant maps of convex bodies the natural domain of definition is $\Re_{0}^{d}$, not $\Re^{d}$.

Proof of $\left(1.4^{\prime}\right)$. In view of the remarks made above, it suffices to show that a continuous, additive map $\Phi: \Omega_{0}^{d} \rightarrow \Omega^{d}$ which commutes with rotations, can be continuously extended to all of $\Re^{d}$ (by continuity, the additivity and equivariance properties carry over to the extension).

It is easy to see that the proof of Lemma (4.7) goes through if $\Phi$ is merely defined on $\AA_{0}^{d}$. We deduce that

$$
\Pi_{m} H_{\Phi K}=\gamma_{m} g_{m} \Pi_{m} H_{K} \quad \text { for each } K \in \AA_{0}^{d}
$$

for $m=0,1,2, \ldots$, where $\gamma_{0}, \gamma_{1}, \ldots$ are suitable real numbers and $g_{0}, g_{1}, \ldots$ are rotations. Let $L \in \Re^{d} \backslash \Omega_{0}^{d}$, and choose a sequence $\left(K_{i}\right)_{i \in \mathrm{N}}$ in $\Omega_{0}^{d}$ such that $\lim K_{i}=L$. By (4.10) and (4.11) we have $s\left(\Phi K_{i}\right)=s\left(K_{i}\right) \rightarrow s(L)$ and $\beta\left(\Phi K_{i}\right)$ $=\gamma_{0} \beta\left(K_{i}\right) \rightarrow \gamma_{0} \beta(L)$ for $i \rightarrow \infty$, hence the sequence $\left(\Phi K_{i}\right)_{i \in \mathrm{N}}$ is bounded and contains, therefore, a convergent subsequence. We may assume $\Phi K_{i} \rightarrow M \in \Re^{d}$ for $i \rightarrow \infty$. By (*) we have

$$
\Pi_{m} H_{M}=\lim \Pi_{m} H_{\Phi K_{i}}=\lim \gamma_{m} g_{m} \Pi_{m} H_{K_{i}}=\gamma_{m} g_{m} \Pi_{m} H_{L}
$$

for $m=0,1,2, \ldots$. This shows that the convex body $M$ is uniquely determined by $L$. We may now define $\Phi L=M$, which gives the desired extension.

\section{REFERENCES}

1. Ch. Berg, Corps convexes et potentiels sphériques, Mat.-Fys. Medd. Danske Vid. Selsk. 37 (1969), no. 6, 64 pp. MR 40 \#7996.

2.—, Abstract Steiner points for convex polytopes, J. London Math. Soc. (2) 4 (1971), 176-180. MR 45 \#7593.

3. W. Blaschke, Kreis und Kugel, 2nd ed., de Gruyter, Berlin, 1956. MR 17, 1123.

4. H. Boerner, Darstellungen von Gruppen. Mit Berücksichtigung der Bedürfnisse der modernen Physik, Zweite, überarbeitete Auflage, Die Grundlehren der math. Wissenschaften, Band 74, Springer-Verlag, Berlin and New York, 1967. MR 37 \#5307.

5. T. Bonnesen and W. Fenchel, Theorie der konvexen Körper, Springer, Berlin, 1934.

6. H. Busemann, Convex surfaces, Interscience Tracts in Pure and Appl. Math., no. 6, Interscience, New York, 1958. MR 21 \# 3900.

7. R. R. Coifman and G. Weiss, Representations of compact groups and spherical harmonics, Enseignement Math. (2) 14 (1968), 121-173. MR 41 \# 537. 
8. R. R. Coifman and G. Weiss, Analyse harmonique non-commutative sur certains espaces homogènes, Lecture Notes in Math., vol. 242, Springer, Berlin and New York, 1971.

9. R. Courant and D. Hilbert, Methods of mathematical physics. Vol. I, Interscience, New York, 1965.

10. C. F. Dunkl, Operators and harmonic analysis on the sphere, Trans. Amer. Math. Soc. 125 (1966), 250-263. MR 34 \# 3224.

11. R. E. Edwards, On convex spans of translates of functions on a group, Proc. London Math. Soc. (3) 3 (1953), 222-242. MR $15,101$.

12. W. Fenchel and B. Jessen, Mengenfunktionen und konvexe Körper, Danske Vid. Selsk. Mat.-Fys. Medd. 16 (1938), no. 3, 31 pp.

13. B. Grünbaum, Measures of symmetry for convex bodies, Proc. Sympos. Pure Math., vol. 7, Amer. Math. Soc., Providence, R.I. 1963, pp. 233-270. MR 27 \#6187.

14. , Convex polytopes, Pure and Appl. Math., vol. 16, Interscience, New York, 1967. MR 37 \#2085.

15. H. Hadwiger, Vorlesungen über Inhalt, Oberfläche und Isoperimetrie, Springer, Berlin, 1957. MR 21 \#1561.

16. H. Hadwiger and R. Schneider, Vektorielle Integralgeometrie, Elem. Math. 26 (1971), 49-57. MR 44 \#967.

17. T. Kubota, Über die konvex-geschlossenen Mannigfaltigkeiten im n-dimensionalen Raume, Sci. Rep. Tôhoku Imperial Univ. 14 (1925), 85-99.

18. W. J. Meyer, Characterization of the Steiner point, Pacific J. Math. 35 (1970), 717-725. MR 43 \#3917.

19. C. Müller, Spherical harmonics, Lecture Notes in Math., vol. 17, Springer-Verlag, Berlin and New York, 1966. MR 33 \# 7593.

20. G. T. Sallee, A non-continuous "Steiner point", Israel J. Math. 10 (1971), 1-5. MR 45 \# 5873.

21. H. Schaal, Prüfung einer Kreisform mit Hohlwinkel und Taster, Elem. Math. 17 (1962), 33-38. MR 25 \# 1489.

22. R. Schneider, On Steiner points of convex bodies, Israel J. Math. 9 (1971), 241-249. MR 43 \#3918.

23.—, Gleitkörper in konvexen Polytopen, J. Reine Angew. Math. 248 (1971), 193-220. MR 43 \#5411.

24.— Krümmungsschwerpunkte konvexer Körper. II, Abh. Math. Sem. Univ. Hamburg 37 (1972), 204-217.

25. G. C. Shephard, Euler-type relations for convex polytopes, Proc. London Math. Soc. (3) 18 (1968), 597-606. MR 38 \#606.

26. - A uniqueness theorem for the Steiner point of a convex region, J. London Math. Soc. 43 (1968), 439-444. MR 37 \#3447.

27. G. Valette, Subadditive affine-invariant transformations of convex bodies, Geometriae Dedicata (to appear).

Fachbereich Mathematik, Technische Universität Berlin, Berlin, Germany

Current address: Mathematisches Institut der Universität, Freiburg i. Br., Germany 